\title{
IPHONE AND IPAD APPLICATIONS: PERCEPTIONS OF USE IN AN ORGANIZATION'S SUPPLY CHAIN
}

\author{
Cassandra C. Elrod, Missouri University of Science and Technology, cassa@mst.edu \\ Chelsea Brookshire, Missouri University of Science and Technology, cebggb@mst.edu \\ Elizabeth A. Cudney, Missouri University of Science and Technology, cudney@mst.edu \\ Barry B. Flachsbart, Missouri University of Science \& Technology, barryf@mst.edu
}

\begin{abstract}
Since 2007, iPhone applications have been utilized in increasing ways in consumer environments, including the workplace. This paper discusses current levels of iPhone and iPad application integration into organizations' daily operations and sought to gather employee knowledge and perceptions of application usage. The potential for greater efficiencies by application integration are also discussed. The data used for this study was gathered using a questionnaire distributed to individuals in varying industries and organizations, from education to military, in order to gain insight into how applications are currently being used, how they could be used, and ways to improve supply chain efficiencies.
\end{abstract}

\section{INTRODUCTION}

In the past decade, technology has dramatically changed everyday routines for most of the industrialized world. Before 2000, most people could not play games or access email via cellular phones. Now, most consumers can perform functions from phones that previously required a computer with an Internet connection. A billion mobile devices will access the Internet in the year of 2010 [1]. This forecast in 2009 has probably been well surpassed.

Technology has redesigned business in past decades. The Internet has made simple business processes, such as remote meetings, digital and cost effective. More recently, a new element has been added to this fast paced business environment: mobility. Throughout the past five years, iPhones and iPads and similar devices have dramatically changed numerous business functions. Smartphone platforms have redefined the business world. The Android/Google system offers consumers a more self-serving system while Blackberry and Apple offer more managed systems. While both types of platforms have been extremely innovative, the iPhone is often recognized for paving the way for this innovation [3]. While the hardware that makes up these devices is cutting edge, there is nothing extremely innovative about the components of iPads or iPhones. The driving factor affecting business and communications is the applications accessed by these devices.

There are hundreds of thousands of iPhone and iPad applications. Apple applications can be categorized in many ways, looking, for example, at the cost of an application or at the supplier of the application. This study found it useful to mix these viewpoints into five main categories: universal, free, paid, subscription, or custom.

The applications, whether created by Apple or an outside entity, allow people from all backgrounds to access sometimes large amounts of information in seconds. Apple's App Store has more than 300,000 applications in various categories [17]. With such a selection, one can often find a free or paid application that is applicable to a specific industry or expertise. For example, there are Six Sigma applications and best practices checklist applications.

No idea is too broad or narrow when it comes to the App Store. Programmers have the option of designing and developing almost any idea into an application [16]. For the right price, programmers will also develop customized applications for a third party. Applications are also created for advertisements or publicity. For instance, authors can create an application for the sole purpose of book promotion. 
In the business world, one area of intense interest is supply chain and all aspects surrounding it. Supply chain innovations can help any company save money. Identifying a potential for more sustainable practices will lead to greater supply chain efficiencies [6]. While considerable research has been conducted in the areas of supply chain, supply chain management, and efficiencies, little research exists in the area of iPhone and iPad applications. This study deployed a questionnaire to individuals in varying industries and organizations, from education to military, in order to gain insight into how applications are currently being used, how they could be used, and ways to improve supply chain efficiencies.

\section{LITERATURE REVIEW}

Launched 30 years ago, mobile telecommunications have grown tremendously and consist of 4.6 billion users and over 1 billion mobile Internet users [7]. Reflecting consumer demands, the mobile phone is evolving into a mobile computing device, functioning like a PC. The average hand held device is designed for a lifecycle of 3.5 years. Rapid replacement is attributed to the tendency of consumers to want a device with more applications and functionality overall.

Smartphones have dramatically changed the way business is conducted and “...have the capacity to increase the amount of information we can consume and distribute, and the times/places where we can do it, adding a valuable level of mobility to the way we work" [5]. Hopkins [5] concludes that smartphones are essential, time saving devices; he also concludes that the majority of business users mainly utilize the email and calendar features of their smartphones.

To be beneficial, the mobile product and service should ensure relevance to the user [19]. Applications of smartphones have taken this concept one step further by adding even more customization options and business solutions. Over the past decade, business has become more mobile and borderless. Applications are particularly convenient to executives that spend more time in planes and cars than in offices. Enterprise applications are created to support business processes from servers in a data center; the applications (on iPhones and iPads and similar devices) empower employees from their device [4]. Empowering employees, historically, leads to higher efficiencies. This empowerment, combined with the speed of mobile workers and mobile applications, could certainly yield improved efficiencies for organizations.

The ability to download applications of nearly any functionality allows iPhone and iPad users to make their device most relevant to their occupation and personal preferences. Flurryanalytics.com [18] illustrates the diversity of iPhone applications and the ability of users to interact with the applications most relevant to their life and providing the most benefit. However, a survey conducted by Flurry Analytics demonstrated that consumers spent the most time using applications for social networking and news. Utility or functional applications were not even considered in the survey; at the time (early 2010), utilizing the functionality of applications on a regular basis was a new concept to many people.

Yet, according to Faulhaber and Farber [3], the application segment has proven extremely innovative. In the past decade the market has grown from a few dozen applications to several hundred thousand. While the race to be the most innovative was in progress before the introduction of the iPhone and iPad, the iPhone raised the bar for all other device makers. Competition in the wireless markets has led to great innovations. The carrier is no longer nearly as important to the consumer; functionality of the device, or its applications, is the innovation [3].

One of the premier industries to take advantage of iPhone applications is the medical field. Custom and universal applications have been used to aid doctors, nurses, and support staff in several areas of medicine. One example is the field of neurological sciences, where studies concluded that the iPhone Teleradiology system accurately detects acute cervicodorsal spinal trauma and allows secure and mobile visualization of medical images; however, no patient data is permanently stored on the phone [10]. Here, the applications, not the phone itself, are used as tools. 
While iPhone applications have made the jobs of medical staff easier, they have also assisted patients. Diabetics use many tools for self-monitoring of blood glucose (SMBG). Studies in this field have shown that sharing SMGB data and feedback from health care providers can help achieve glycemic goals. "Being able to record, analyze, seamlessly share, and obtain feedback on the SMBG data using an iPhone/iTouch application might potentially benefit patients. Trends in SMBG data management and the possibility of having interoperability of blood glucose monitors and smartphones may open up new avenues of diabetes management for the technologically savvy patient” [12]. Applications can improve efficiency while at the same time improve customer care and service. Electronic health records have proven to be innovative and life saving; adding the factor of mobility to this and similar innovations only further increases efficiencies, leading to more effective patient care. Having increasingly accurate and mobile health records and information greatly reduces error and improves, even saves, the lives of patients; this is a major innovation for the medical industry. Using an iPhone for diagnostics and imaging is highly innovative [14].

Mobile devices are also being utilized in Location-Based Logistic Services to assist employees. Mobile devices support the order execution process. They route the deliverers to their next destination and keep them informed about past and future orders. Mobile devices enable the tracking of the current order state, the actual position of the packages, and the employee's position for extending the existing order disposition and routing processes [2].

Numerous studies have been conducted in regards to supply chain, supply chain management, and efficiencies. Traditionally, supply chains are decentralized and each segment is evaluated individually; this approach often leads to poor performance of the system [9]. Centralization, integration, and communication are crucial to maximizing efficiencies of the supply chain, regardless of the industry. Supply chain managers can reach success by collaborating closely with suppliers [15]. Case studies from the mobile phone industry on Apple and Android platforms illustrate that innovation initiatives can approach generativity in different ways and control and openness are important to facilitate supplier contributions [13]. Additional mobile practices may be able to enhance collaboration and integration.

A common goal of supply chain managers is to reduce mistakes and improve efficiencies; this can be done by increasing employee productivity, saving time and other resources, and integrating new technologies. Production and scheduling problems often lead to inefficiencies. Supply chain planning and production scheduling problems are similar in nature. Both seek solutions to questions of amounts, when, and where to produce. Problems also occur when attempting to answer and provide solutions to these questions while maintaining a healthy financial portfolio for the company [11]. Being able to come to solutions faster and obtain real-time data would certainly be helpful to the process. Adding more mobility to the production and scheduling processes could help eliminate errors.

\section{METHODOLOGY}

A questionnaire was drafted to obtain knowledge about perceptions, relevance, and implementations of iPhone and iPad applications, with specific questions relating to supply chain. The goal was to answer questions such as: "What do industry employees know about applications?" "Are employees aware of the benefits?" "How many managers are implementing the use of applications in the work environment?" Respondents of a broad range of service and manufacturing industries were solicited in order to seek answers to questions about which industries are implementing applications.

The questionnaire was designed to gather the respondents' perceptions of applications before asking questions regarding the supply chain. If the respondent implemented applications in the work environment, further questions were asked to gather information about the results of implementation. iPhones and iPads are the most popular and fastest growing mobile devices; the Apple App Store has more applications than all other application providers combined; thus, the scope of the questionnaire is on Apple applications exclusively. The questionnaire was distributed online via Qualtrics.com to individuals in various industries. Incomplete questionnaire responses were removed from the analysis. This paper includes responses from 36 individuals in different industries. 


\section{RESULTS}

Figure 1 shows the percent of respondents in each industry represented in the respondent pool. It is clear from Figure 1 that nearly half of respondents are in Education or "Other" industries. Presently, there are many mixed studies, opinions, and regulations in regards to the cost, boundaries, and technologies used in the field of education.

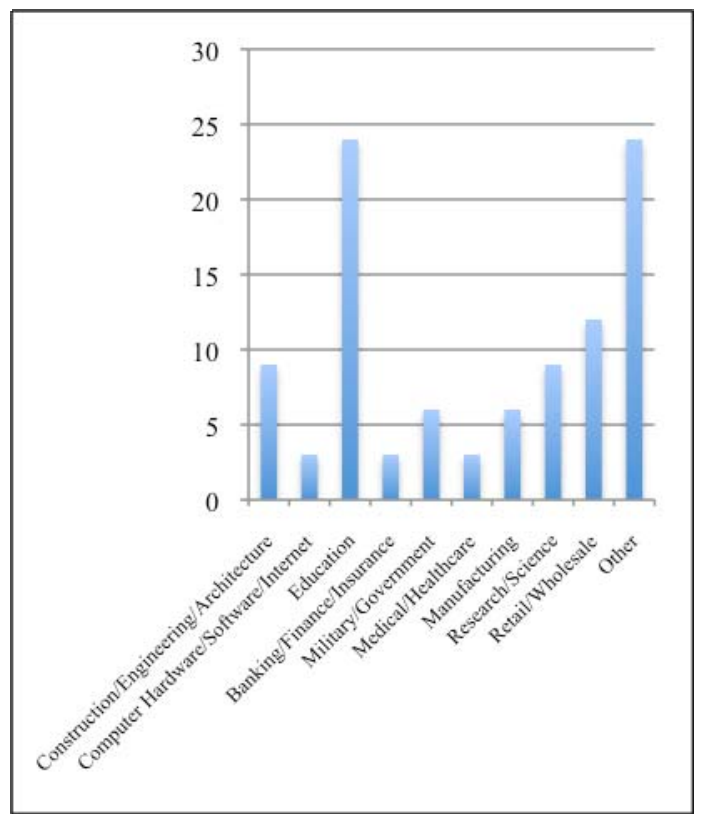

Figure 1: Percent of total respondents per industry

Supply chain operations are important to all companies, whether manufacturers or service providers. Figure 2 illustrates the primary function of the organizations in which respondents are employed. As shown, the service industry was the type of organization where the majority of the respondents were employed.

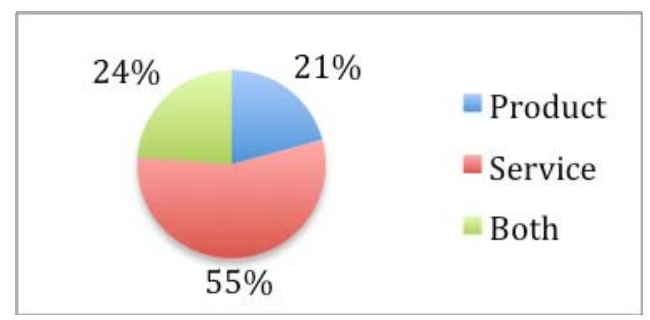

Figure 2: Respondent’s company function

Figure 3 illustrates the company size in which the respondents were employed. It is clear that respondents are relatively evenly divided; slightly more than half are employed at organizations with less than 1,000 employees and the remainders are employed at organizations with over 1,000 employees. 
Volume XII, No. 1, pp. 170-180, 2011

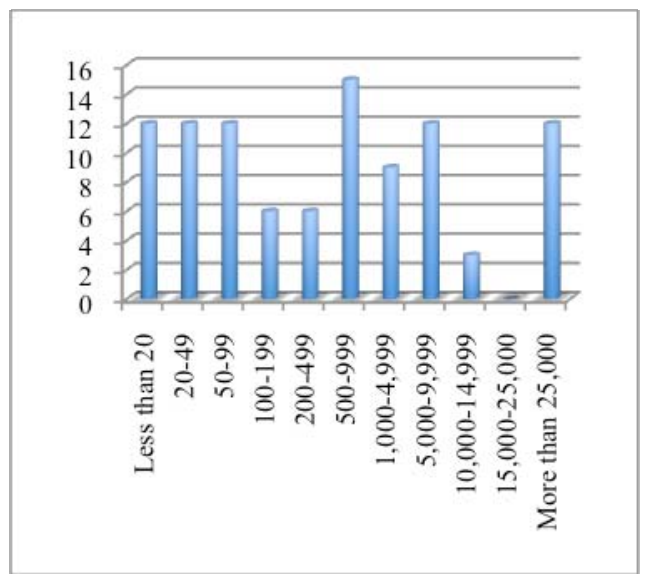

Figure 3: Company Size (\% of respondents)

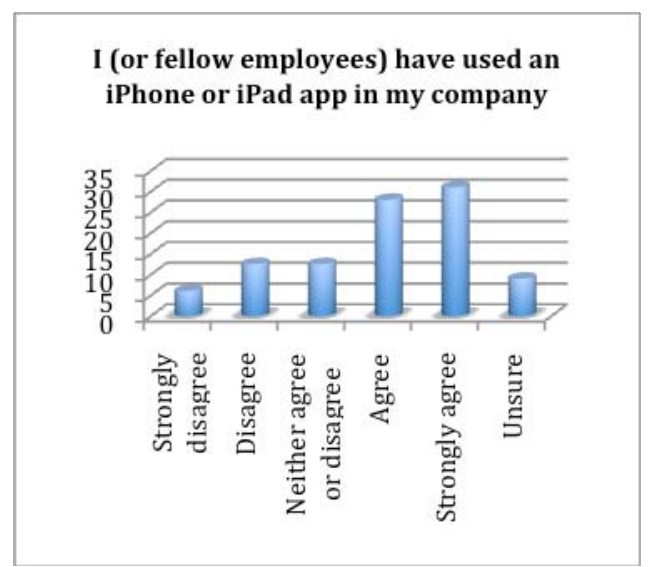

Figure 4: Respondent's use of applications in the workplace

Of the respondents, $44 \%$ stated that iPhone and/or iPad applications are used at their company. This is illustrated in Figure 5.

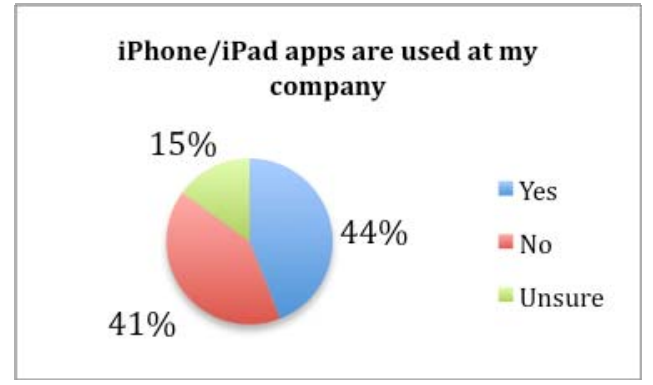

Figure 5: Percentage of companies in industry currently using iPhone/iPad applications

Of the $44 \%$ that use iPhone/iPad applications in industry, the breakdown by classification of the applications used is illustrated in Figure 6. 
Volume XII, No. 1, pp. 170-180, 2011

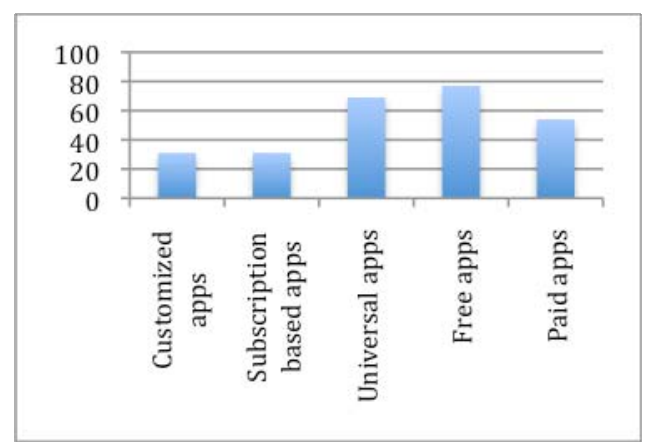

Figure 6: Types of applications used in industry

General information gathered from the questionnaire resulted in information regarding respondents' perceptions regarding efficiencies and types of information applications can be useful for in organizations. For example, respondents were asked a series of questions to gather perceptions of iPhone and iPad applications in regard to supply chain use in their organization. First, they were asked about the statement: "Improved businesses practices can lead to higher efficiencies." Seventy-five percent of respondents agreed or strongly agreed that applications can improve business practices. Further, "Applications have the ability to provide real time information to business people or a company database" was presented. Sixty percent of respondents agreed or strongly agreed with this concept, 38\% were neutral or unsure, and only one respondent disagreed. This shows that while a majority is familiar with the capabilities of iPhone and iPad applications, there are still a significant number of business people not aware of application functions and their capabilities.

It is also important to understand industry perceptions about how iPhone and iPad applications affect the supply chain in general. Figure 7 illustrates the percent of respondents that agreed or disagreed with the concepts of sustainability and enhancement of the supply chain via applications.

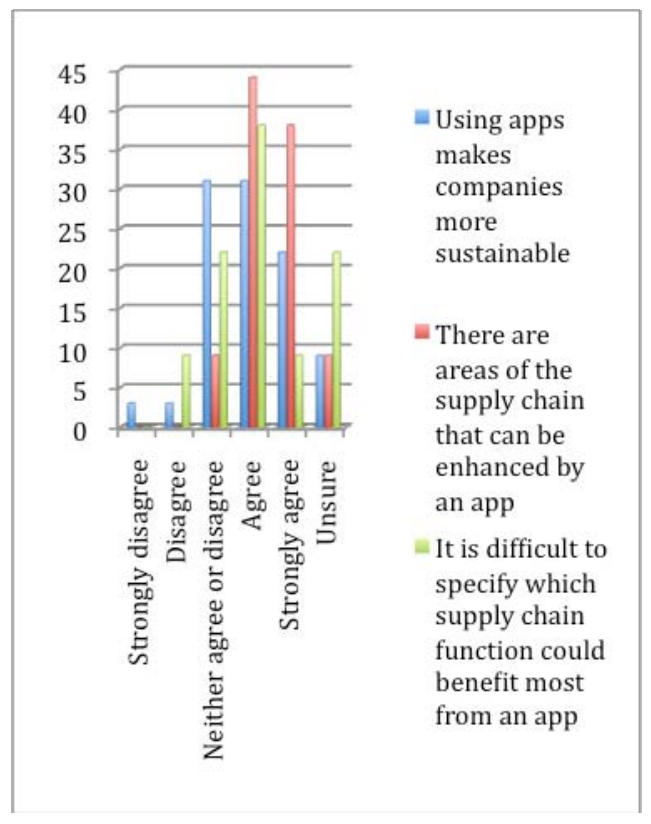

Figure 7: Respondent's perceptions of sustainability and enhancement 
Improving supply chain efficiencies can contribute to a competitive advantage. Figure 8 demonstrates respondent's perceptions of using different kinds of applications to stay competitive. A majority of respondents feel that companies should use applications to stay competitive, with a clear preference by respondents for customized applications over universal or subscription applications.

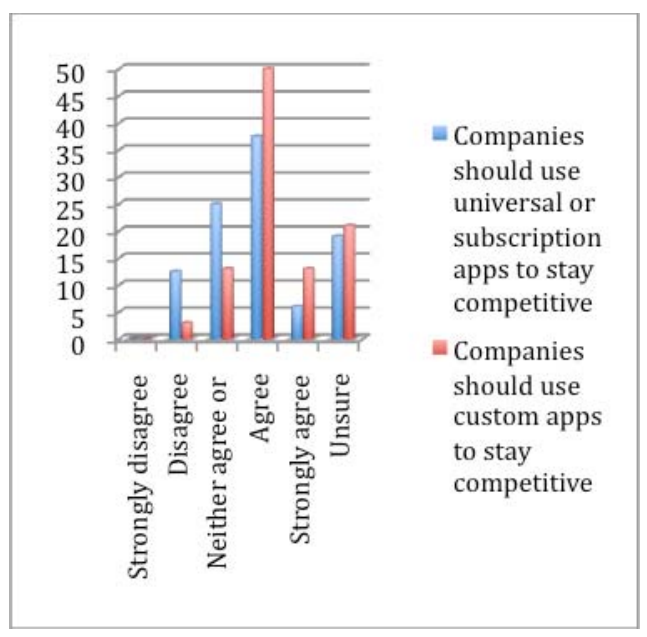

Figure 8: Respondent’s perceptions of competitiveness

Related to staying competitive, respondents were also asked if they believed universal or custom applications could save their company money. Figure 9 shows these perceptions. Again, while a majority of respondents agree, a significant number are neutral or unsure. Figures 8 and 9 show that respondents agree with both concepts of applications helping competitiveness and saving money; however, they are more confident in the idea of applications saving money than in applications helping competitive advantage.

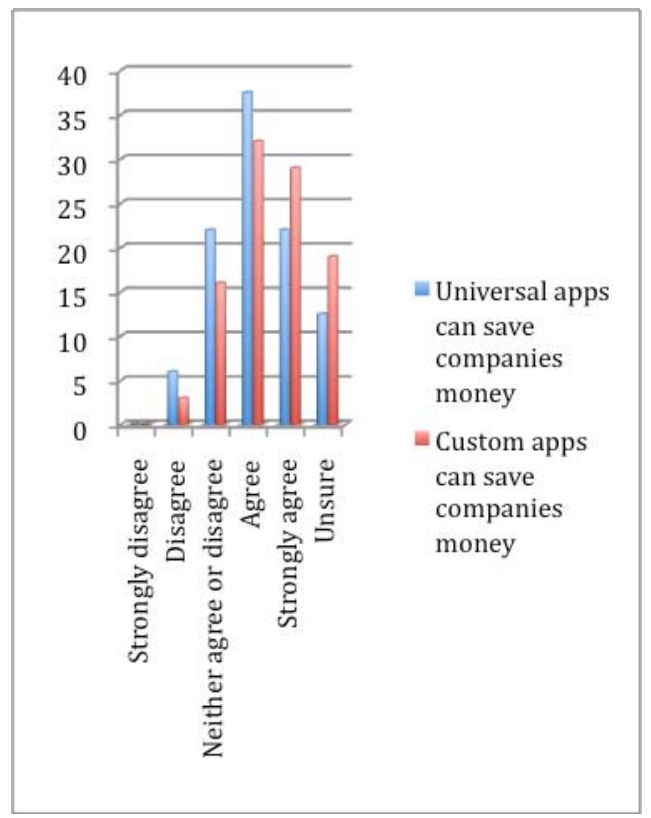

Figure 9: Respondent’s perceptions of iPhone and iPad applications' ability to save money 
Figure 10 illustrates respondents' perceptions of the effect of applications on productivity. Almost everyone agreed with the idea that productivity can be positively affected by applications.

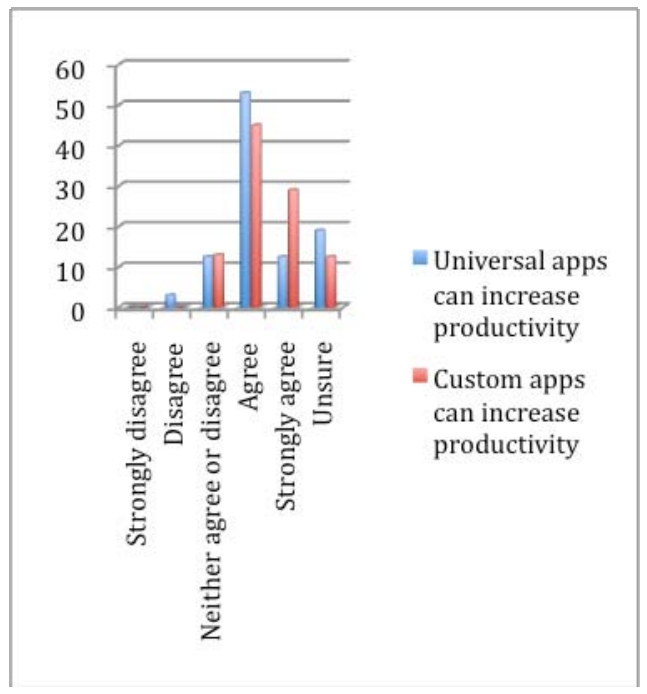

Figure 10: Respondent's perceptions of iPhone and iPad applications' ability to increase productivity

To gain some insight into user perceptions, a word association methodology was used to ask respondents to list the first three words that come to mind when asked about iPhone or iPad applications (Kotler, 2003). All responses were grouped into the categories shown in Figure 11. The first column represents the first response of the participant and its category; the last column represents the total occurrence of words in each category from all three responses. Of respondents, $24 \%$ firstly associated applications with a word that illustrated the benefits or functionality of iPhone or iPad applications, such as "instant," "time," "helpful," and "convenience." Nearly 20\% think of phones or Apple first, not the actually functionality or benefits of applications.

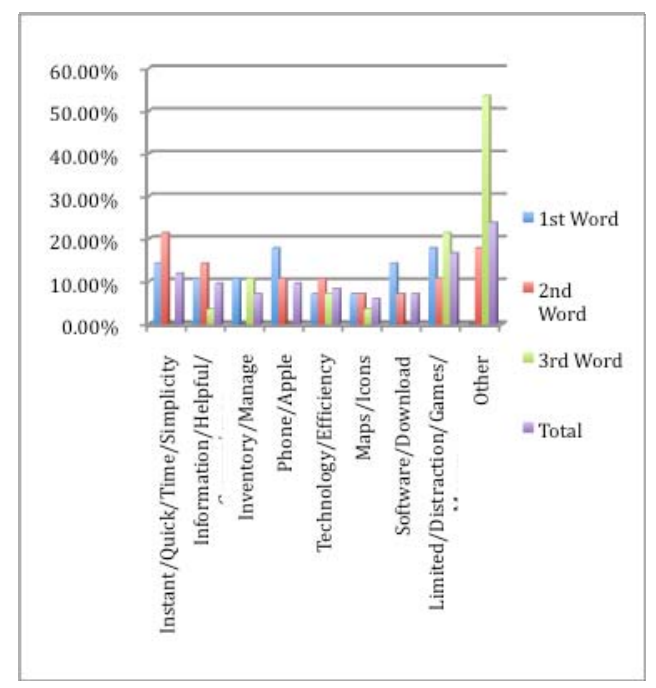

Figure 11: Responses in word association of "iPhone \& iPad applications" 
The respondents that implemented applications of all varieties in the business place were asked their perceptions of the effectiveness of the implementations. While a majority agreed that there are benefits of app implementation, such as operations efficiency, there are still many that are neutral or unsure. This is represented in Figure 12. Of respondents, $70 \%$ agree an increase in productivity is seen. But only half agree that elements of the supply chain have become more efficient. It is important to note that half of respondents do not disagree but are neutral or unsure of the application's effect on supply chain efficiencies.

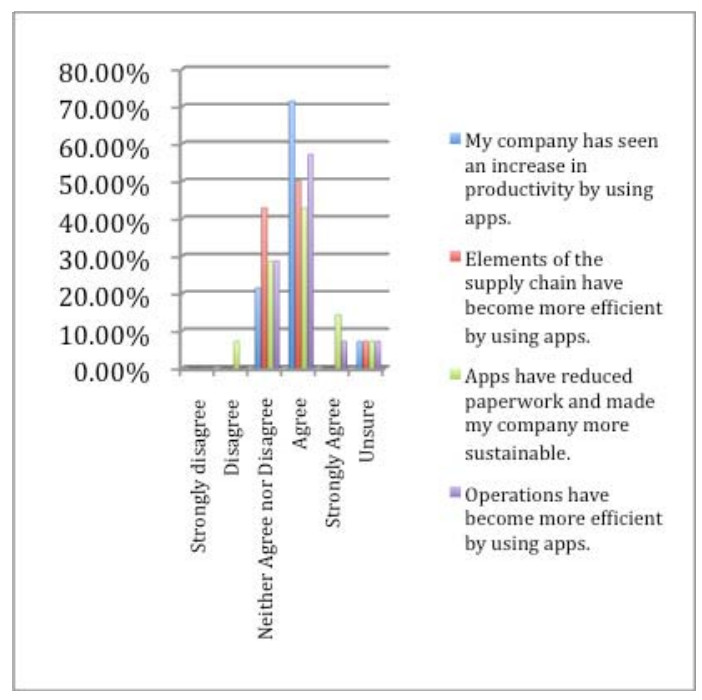

Figure 12: Respondent's perceptions of iPhone application implementation at the work place

Lastly, respondents were given the opportunity to provide remarks in regards to iPhone and iPad applications and implementations and implications in industry. Most of the remarks dealt with seeing or anticipating positive, beneficial information and efficiencies as a result of integrating applications into the supply chain. The comments received are provided in Table 1.

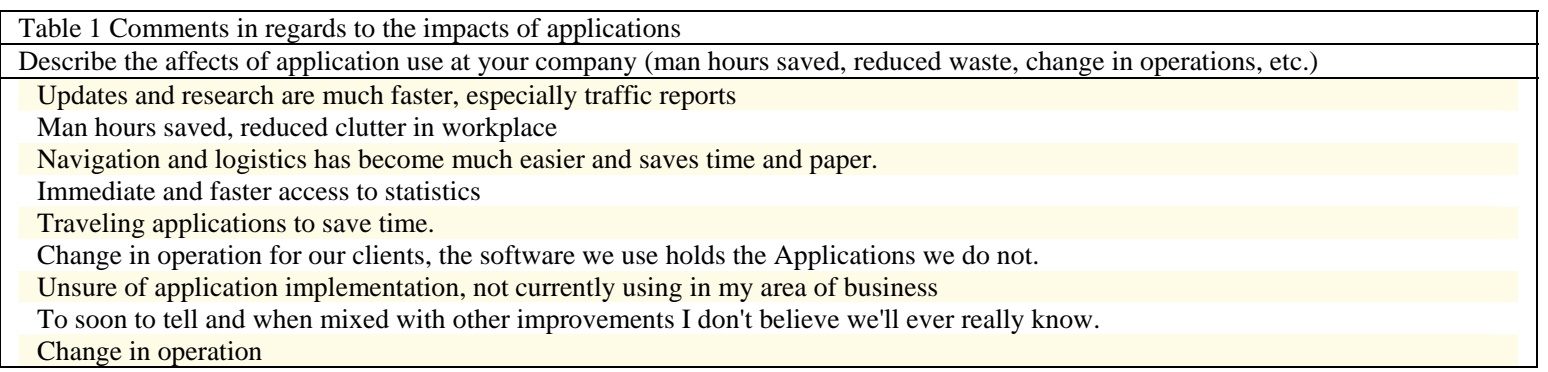

\section{CONCLUSION}

The perceptions of iPhone and iPad applications vary greatly among industry employees. While some fully understand and utilize the benefits, others are skeptical or simply unaware of any advantages. While consumers may not be fully aware of all the capabilities of applications, there seems to be some evidence that respondents see that applications can be of value in an organization's operations and supply chain.

Nearly half of respondents in industry are using applications in some form at the workplace. This concludes that in just a few short years, a new factor has been added to the operations of various types of 
organizations. A majority of employees using applications claim improved efficiencies, such as reduction in paperwork.

The applications of iPhones and iPads allow employees of all levels to perform faster and more efficiently. The fact that slightly over half of respondents are not using applications or are unsure if their company is using applications, illustrates that this is a very new innovation and that applications have not been heavily implemented in all types of organizations. The respondents that use applications agree that the implementation has been innovative. Saving time, money, waste, and increasing productivity with the tap of a screen are promising improvements.

It might be assumed that iPhone and iPad applications would be powerful aids to daily operations and trickle down into the supply chain. Paper and time can be saved. Extensive calculations and navigation can be done in seconds. However, it is clear that less than half of respondents use applications in the workplace in any way. Of the companies that do utilize the benefits of applications, most do not have a clear understanding of the benefits or use the full potential that applications might provide. Downloads and awareness of applications and their functions has grown at a tremendous rate in the four years since their first unveiling. As more and more consumers and developers utilize and create applications with even more useful functionalities, the impacts for operations and supply chain are likely to be further clarified.

\section{FUTURE RESEARCH}

A more extensive questionnaire should be performed to inquire exactly where, when, and how managers would utilize applications in the supply chain. One might also ask about what activities in supply chain work might most benefit from potential applications. This could aid in the development of a framework to guide companies to utilizing applications to their full potential in improving supply chain efficiencies.

\section{REFERENCES}

1. Elmer-Dewitt, P. (2009). IDC: 300,000 Applications by 2011. Apple 2.0. Retrieved 21 October from, http://tech.fortune.cnn.com/2009/12/03/idc-300000-iphone-applications-by-2011/

2. Emmersberger, C., Springer, F., and Wolff, C. (2009). Location Based Logistics Services and Event Driven Business Process Management. University of Regensburg, $9^{\text {th }}$ Expert Meeting 08 November 2009. CCIS, 53: 167-177.

3. Faulhaber, G.R. and Farber, D.J. (2010). Innovation in the Wireless Ecosystem: A CustomerCentric Framework. International Journal of Communication, 4: 73-112.

4. Gonzalez, A. (2009). Software for the Masses: The iPhone, Crowdsourcing and Logistics. Retrieved 18 October, 2010 from, http://logisticsviewpoints.com/2009/09/30/software-for-the-massesthe-iphone.

5. Hopkins, J. (2010).Smartphones tune into the Supply Chain. POMS $21^{\text {st }}$ Annual Conference, Vancouver, Canada.

6. Isaksson, R., Johansson, P., and Fischer, K. (2010). Detecting Supply Chain Innovation Potential for Sustainable Development. Journal of Business Ethics.97: 425-442.

7. Jacobson, M., Niemegeers, I., and Groot, S. (2010). Personal Networks: Wireless Networks for Personal Devices. John Wiley \& Sons Ltd. 1-4.

8. Kotler, P. (2003). Marketing Management. Upper Saddle River, New Jersey, Prentice Hall.

9. Mestan, E., Turkay M., and Arkun, Y. (2006). Optimization of Operations in Supply Chain Systems Using Hybrid Systmes Approach and Model Predictive Control. American Chemical Society. 45: 64936503.

10. Modi, J., Sharma, P., Earl, A., Simpson, M., Mitchell, J., and Goyal, M. (2010). iPhone-Based Teleradiology for the Diagnosis of Acute Cervico-Dorsal Spine Trauma. The Canadian Journal of Neurological Sciences 37 (6).

11. Puigjaner, L., Lainez, J., and Alvarez, C. (2009). Tracking the Dynamics of the Supply Chain for Enhanced Production. American Chemical Society, 48: 9556-9570.

12. Rao, A., Hou, P., Golnik, T., Flaherty, J., and Vu, S. (2010). Evolution of data management tools for 
managing self-monitoring of blood glucose results: a survey of iPhone Applications. Journal of Diabetes Science \& Technology 4 (4) 958-960.

13. Remneland, B., Ljungberg, J., and Bergquist, M. (2011). Open Innovation, Generativity and the supplier as peer: The case of Apple a and Android. IJIM 15, 1 pp. 205-230.

14. Shih, G., Lakhani, P., and Nagy, P. (2010. Is Android or iPhone the Platform for Innovation in Imaging Informatics. Journal of Digital Imaging 23, 1 pp. 2-7.

15. Tarigan, Z. (2009). The Impact of Strategic Purchasing to the Supplier Involvement through Communication, Coordination and Collaboration in Small and Medium Enterprises. Proceeding, International Seminar on Industrial Engineering and Management. Inna Kuta Beach, Bali.

16. Want, R. (2010). iPhone: Smarter Than the Average Phone. Pervasive Computing, IEEE 9, 3, pp. 6-9.

17. www.apple.com

18. www.flurryanalytics.com

19. Zadok, G., and Puustinen, R. (2010). The Green Switch: Designing for Sustainability in Mobile Computing. Proceedings of the First USENIX conference on Sustainable Information Technology. San Jose, CA. 